\title{
ISOSPIN CHARACTER OF LOW-LYING PYGMY DIPOLE STATES PROBED VIA INELASTIC SCATTERING OF ${ }^{17} \mathrm{O}^{*}$
}

\author{
F.C.L. Crespi, A. Bracco, L. Pellegri \\ Dipartimento di Fisica dell'Università degli Studi di Milano and INFN \\ 20133 Milano, Italy
}

(Received January 21, 2015)

\begin{abstract}
The pygmy dipole states were populated in ${ }^{208} \mathrm{~Pb}$ and ${ }^{124} \mathrm{Sn}$ by the inelastic scattering of a ${ }^{17} \mathrm{O}$ beam at the energy of $20 \mathrm{MeV} / u$, and their subsequent gamma decay was measured with the AGATA demonstrator array. Differential cross sections as a function of the angle were measured. The results are compared with $\left(\gamma, \gamma^{\prime}\right)$ data. For the dipole transitions, a form factor obtained by folding a microscopically calculated transition density was used. This has allowed us to extract the isoscalar component of the $1^{-}$excited states.
\end{abstract}

DOI:10.5506/APhysPolB.46.501

PACS numbers: 24.30.Cz, 24.10.Eq, 25.55.Ci, 27.80. $+\mathrm{w}$

\section{Introduction}

The electric dipole (E1) strength in atomic nuclei is almost completely concentrated in the extensively studied isovector giant dipole resonance (IVGDR). For neutron-rich nuclei, the E1 response is characterized by concentrations of strength, denoted as pygmy dipole resonance (PDR) or pygmy states, around and below the particle separation energy [1]. The PDR is expected to reflect the properties of the neutron skin [2-8] and this is used to constrain the equation of state of neutron-rich matter. One important open problem for pygmy states is the cross section sensitivity to transition densities containing the nuclear structure information. The pygmy states are characterized by transition densities with peculiar features: neutron and proton are in phase inside the nucleus, while at the surface only the neutron part survives. To explore this, one needs high-resolution measurements and the comparison of data obtained with different probes. In particular, it is interesting to use a probe interacting mainly at the surface of the nucleus. This is the case of ${ }^{17} \mathrm{O}$ ions at the energy of $20 \mathrm{MeV} / u$.

* Presented at the Zakopane Conference on Nuclear Physics "Extremes of the Nuclear Landscape", Zakopane, Poland, August 31-September 7, 2014. 


\section{The experiment}

We performed inelastic scattering experiments using a ${ }^{17} \mathrm{O}$ beam at the energy of $20 \mathrm{MeV} / u$ provided by Tadem-ALPI accelerator complex at the LNL-INFN laboratory. Different target nuclei were studied, but we concentrate here on the cases of ${ }^{208} \mathrm{~Pb}$ and ${ }^{124} \mathrm{Sn}$. The scattered ${ }^{17} \mathrm{O}$ ions were detected using two segmented silicon $\Delta E-E$ telescopes $[9,10]$ that were placed symmetrically with respect to the beam direction. These detectors have an energy resolution of about $0.3 \%$ at $340 \mathrm{MeV}$ allowing a good separation of the oxygen isotopes. The gamma decay of the pygmy dipole states was measured with high resolution in coincidence using the AGATA demonstrator [11]. In Fig. 1 the AGATA gamma spectrum, for the case of the ${ }^{208} \mathrm{~Pb}$ target, is shown in the $4.5-8 \mathrm{MeV}$ range. This spectrum is obtained requesting the ${ }^{17} \mathrm{O}$ inelastic scattering channel and that the energy detected in AGATA be equal to the Total Kinetic Energy Loss (TKEL) in the silicon detectors, this latter condition allows to select transitions to the ground state. Furthermore, the spectrum has been Doppler corrected for the recoil velocity. In fact, since the pygmy states have a lifetime of the order of $1 \mathrm{fs}$ or shorter, the gamma rays are emitted when the target nucleus is still recoiling. Furthermore, an unfolding procedure [12] has been applied to the spectrum to suppress the escape peaks and most of the Compton background. In the energy region of the E1 pygmy states also E2 transitions are present. In order to obtain a spectrum in which the E1 transitions we are interested in are enhanced, we applied an additional gating condition at the angle between the direction of the recoil and of the emitted gamma ray. The selected angular range is $65^{\circ}-115^{\circ}$, corresponding to the region in which the E1 multipolarity is maximized.

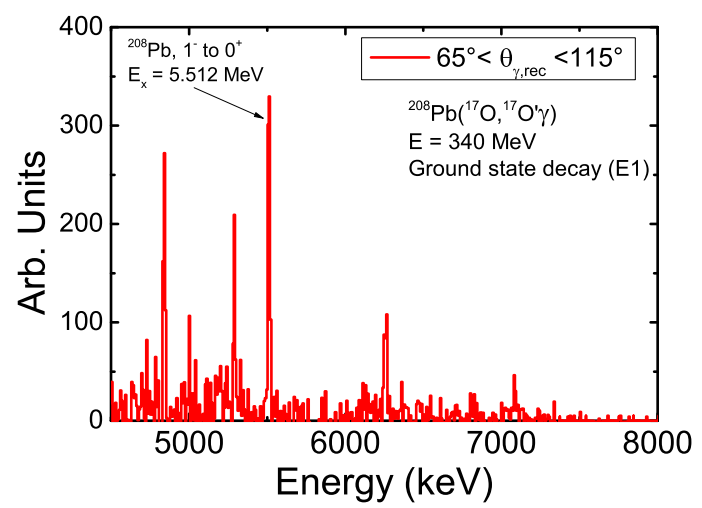

Fig. 1. Gamma-ray spectrum obtained with the AGATA array displayed in the 4.5-8 $\mathrm{MeV}$ region, with the additional condition at the angle between the emitted gamma ray and the recoil direction which enhances the E1 component. 


\section{Results}

The measured angular distributions of the scattered ${ }^{17} \mathrm{O}$ ions were analyzed performing DWBA calculations using the code FRESCO [13]. The first step was to calculate the ${ }^{17} \mathrm{O}+{ }^{208} \mathrm{~Pb}$ elastic scattering cross section. This optical model calculations permitted to determine the absolute normalization of the data. The upper panel of Fig. 2 shows the data for elastic scattering divided by the Rutherford cross section. The optical model parameters of Saxon-Woods potentials providing the best fit to the data used correspond to $V=40.0 \mathrm{MeV}, W=42.5 \mathrm{MeV}$ (with $V$ and $W$ the depth of the real and imaginary potentials), $r_{v}=r_{W}=1.15 \mathrm{fm}, a_{V}=a_{W}=0.767 \mathrm{fm}$ (the radii and diffuseness of the real and imaginary parts) and $r_{\mathrm{C}}=1.20 \mathrm{fm}$ (the Coulomb radius parameter). These are consistent with previous measurements at similar energy $[14,15]$. The differential cross section was determined for the $3^{-}$at $2613 \mathrm{keV}$ and it is shown in the bottom panel of Fig. 2. The experimental data are compared with DWBA calculations performed using the optical model potential parameters determined from the

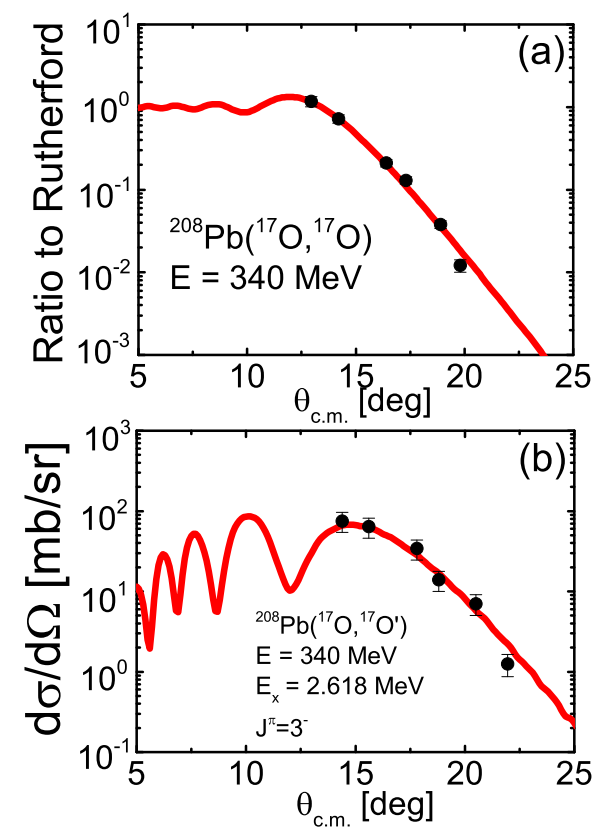

Fig. 2. Experimental cross sections (filled circles) and DWBA calculations (solid curves) for the elastic and inelastic scattering of ${ }^{17} \mathrm{O}$ on ${ }^{208} \mathrm{~Pb}$ at $E_{\text {beam }}=340 \mathrm{MeV}$. Upper panel: Elastic-scattering cross section divided by the Rutherford cross section. Bottom panel: cross section for the $3^{-}$state at $2.618 \mathrm{MeV}$. Figure adapted from [17]. 
${ }^{17} \mathrm{O}+{ }^{208} \mathrm{~Pb}$ elastic data. In these calculation, the adopted $B(\mathrm{E} 3) \uparrow$ is used as input for the code. Moreover, pure isoscalar excitation is assumed, implying that the ratio of the neutron matrix element and the proton matrix element is given by $M_{n} / M_{p}=N / Z$. It is evident that the calculations are able to reproduce well the measured differential cross section.

The DWBA calculations for the differential cross section of the pygmy states obtained using the same procedure adopted for the $3^{-}$state are in a strong disagreement with the experimental data. The result of this calculation using the standard collective model form factor for the nuclear part and the $B(\mathrm{E} 1) \uparrow$ value from $\left(\gamma, \gamma^{\prime}\right)$ measurements is shown with the dotdashed/green line in the left panel of Fig. 3. In particular, in the figure, it can be seen from the comparison with the experimental data that only a fraction of the measured yield is accounted for. In order to make more realistic calculations for the pygmy dipole states, a microscopic form factor was obtained for ${ }^{17} \mathrm{O}+{ }^{208} \mathrm{~Pb}$, by using a double folding procedure with an M3Y nucleon-nucleon interaction [16]. This form factor is derived from the transition density that is shown in the right panel of Fig. 3. The DWBA calculation performed using this form factor for the nuclear part allows to reproduce well the data. Using this procedure, it was also possible to extract the value of isoscalar dipole (ISD) EWSR associated to the pygmy states.
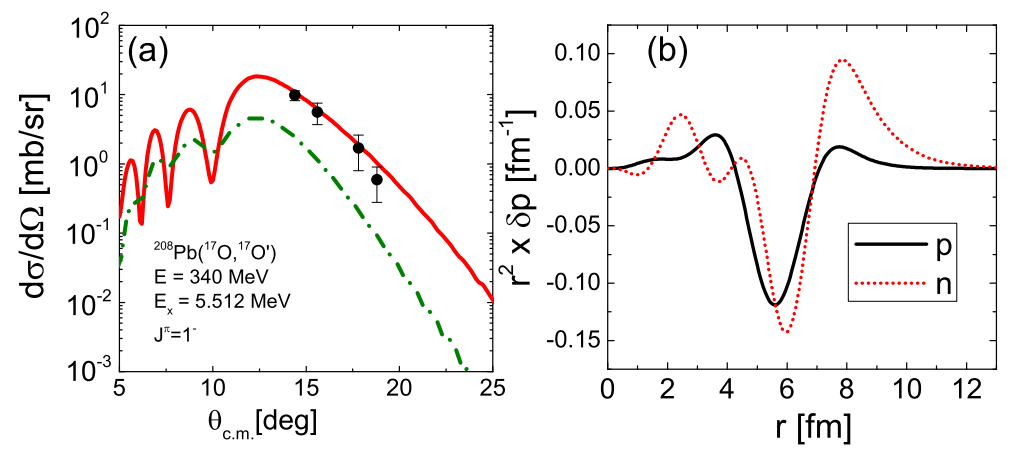

Fig. 3. Left panel: Differential cross section for the $1^{-}$state at $5.512 \mathrm{MeV}$. The lines show DWBA calculations. The dot-dashed/green curve represent the calculation with the standard phenomenological form factor, while the solid/red line represent the calculation with the microscopic form factor for the pygmy states. Right panel: transition density associated to the PDR states for the ${ }^{208} \mathrm{~Pb}$. Figure adapted from [17].

The fraction of the exhausted ISD EWSR for the pygmy dipole states derived from this $\left({ }^{17} \mathrm{O},{ }^{17} \mathrm{O}^{\prime} \gamma\right)$ experiment [17] is shown in Fig. 4 in comparison to the $B(\mathrm{E} 1) \uparrow$ values from $\left(\gamma, \gamma^{\prime}\right)$ experiment on ${ }^{208} \mathrm{~Pb}$ [18]. The figure shows a selectivity in the population of the pygmy states as compared 
to photon scattering results. The observed behavior is similar to what was found in other nuclei using the $\left(\alpha, \alpha^{\prime} \gamma\right)$ reaction [19, 20], a number of states concentrated in the lower energy part of the spectrum are better populated via $\left({ }^{17} \mathrm{O},{ }^{17} \mathrm{O}^{\prime} \gamma\right)$, while the higher lying group of states of isovector nature are suppressed in the isoscalar channel.

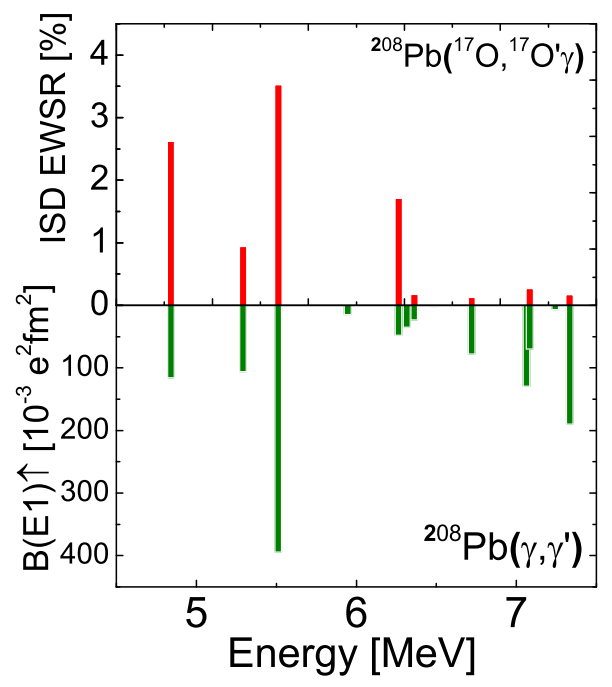

Fig. 4. Upper panel: fraction of the exhausted ISD EWSR for the pygmy dipole states derived from $\left({ }^{17} \mathrm{O},{ }^{17} \mathrm{O}^{\prime} \gamma\right)$ experiment and $B(\mathrm{E} 1) \uparrow$ values from $\left(\gamma, \gamma^{\prime}\right)$ experiment on ${ }^{208} \mathrm{~Pb}$ [18] (bottom panel).

The same kind of DWBA analysis has been applied to the data acquired with ${ }^{124} \mathrm{Sn}$ target. Also in this case, the comparison of the measured cross section of $\left({ }^{17} \mathrm{O},{ }^{17} \mathrm{O}^{\prime} \gamma\right)$ [21] with $\left(\gamma, \gamma^{\prime}\right)$ data evidenced the splitting of the PDR states in two regions as in the $\left(\alpha, \alpha^{\prime} \gamma\right)$ experiments: a low lying part of the E1 strength characterized by isoscalar transition densisities that are peaked on the surface which lead to an enhancement in the isoscalar E1 response, while the higher-lying states can be interpreted as transitions towards the GDR [20]. The first step of the DWBA analysis consisted in the calculation of the elastic scattering cross section, which allowed to extract an overall normalization factor related to the beam current and target thickness. This normalization factor was used for every other measured inelastic scattering cross section. The calculations for different $2^{+}$excited states performed using the standard collective form factor and the adopted $B(\mathrm{E} 2) \uparrow[22,23]$ were able to reproduce well the experimental data. For the $1^{-}$pygmy states, a calculation including both the Coulomb and nuclear contributions and corresponding to a form factor of GDR type is shown in Fig. 5 (solid/green line). The calculated curve lies significantly below the 
experimental data. In order to reproduce the measured cross section, similarly to what was done for the case of ${ }^{208} \mathrm{~Pb}$, another DWBA calculation was performed (dotted/red line) using a microscopic form factor based on the transition density associated to the E1 pygmy states. This calculation was fitted to the experimental data and this allowed to extract the value of the ISD EWSR which resulted to be $1.5(0.2) \%$ for the sum of the measured discrete states in the interval $5.5-7 \mathrm{MeV}$.

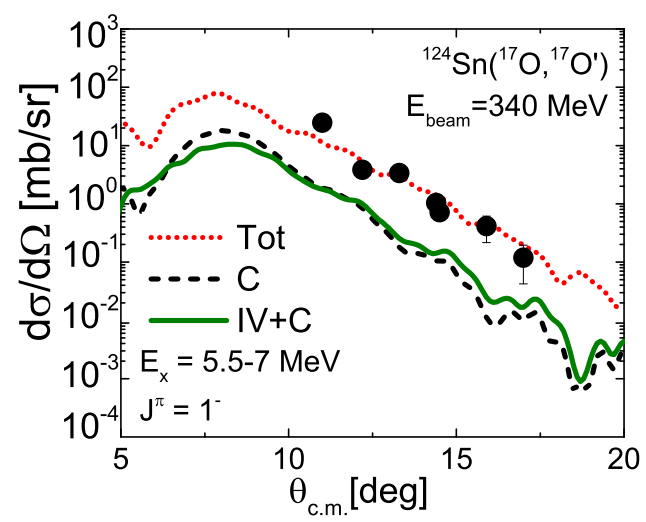

Fig. 5. Differential cross sections for the $1^{-}$states between 5.5 and $7 \mathrm{MeV}$. Dashed/black line: calculation showing the Coulomb contribution; solid/green line: calculation including both the Coulomb and nuclear contributions with a form factor of GDR type; dotted/red line: calculation which uses a microscopic form factor based on the transition density associated to the E1 pygmy states. Figure adapted from [21].

\section{Conclusions}

The inelastic scattering of the ${ }^{17} \mathrm{O}$ ions at $20 \mathrm{MeV} / u$ has been used to populate the pygmy states in ${ }^{208} \mathrm{~Pb}$ and ${ }^{124} \mathrm{Sn}$ nuclei. The DWBA analysis allowed to reproduce the differential cross sections for elastic scattering and for inelastic excitation of the $2^{+}$and $3^{-}$states. The differential cross section for the dipole transition was reproduced using a form factor obtained by folding a microscopically calculated transition density associated to the pygmy states. This allowed to extract the isoscalar component of the $1^{-}$ excited states.

\section{REFERENCES}

[1] D. Savran, T. Aumann, A. Zilges, Prog. Part. Nucl. Phys. 70, 210 (2013).

[2] A. Klimkiewicz et al., Phys. Rev. C76, 051603 (2007).

[3] A. Carbone et al., Phys. Rev. C81, 041301(R) (2010).

[4] O. Wieland et al., Phys. Rev. Lett. 102, 092502 (2009). 
[5] A. Tamii et al., Phys. Rev. Lett. 107, 062502 (2011).

[6] X. Roca-Maza et al., Phys. Rev. C85, 024601 (2012).

[7] X. Viñas, M. Centelles, X. Roca-Maza, M. Warda, Eur. Phys. J. A50, 27 (2014).

[8] N. Paar, D. Vretenar, E. Khan, G. Coló, Rep. Prog. Phys. 70, 691 (2007).

[9] D. Mengoni et al., Nucl. Instrum. Methods Phys. Res. A764, 241 (2014).

[10] A. Gadea et al., Nucl. Instrum. Methods Phys. Res. A654, 88 (2011).

[11] S. Akkoyun et al., Nucl. Instrum. Methods Phys. Res. A668, 26 (2012).

[12] D.C. Radford et al., Nucl. Instrum. Methods Phys. Res. A258, 111 (1987).

[13] I.J. Thompson, Comput. Phys. Rep. 7, 167 (1988).

[14] J.R. Beene et al., Phys. Rev. C39, 1307 (1989).

[15] D.J. Horen et al., Phys. Rev. C44, 128 (1991).

[16] E. Lanza et al., Phys. Rev. C84, 064602 (2011).

[17] F.C.L. Crespi et al., Phys. Rev. Lett. 113, 012501 (2014).

[18] T. Shizuma et al., Phys. Rev. C78, 061303 (2008).

[19] D. Savran et al., Phys. Rev. Lett. 100, 232501 (2008).

[20] J. Endres et al., Phys. Rev. Lett. 105, 212503 (2010).

[21] L. Pellegri et al., Phys. Lett. B738, 519 (2014).

[22] K. Govaert et al., Phys. Rev. C57, 2229 (1998).

[23] H. Iimura et al., Nucl. Data Sheets 80, 895 (1997). 